\title{
Nursing care for a patient with right frontoparietal inflammatory granuloma complicated with acute pulmonary embolism: a case report
}

\author{
Jing Zhang", Yeping $\mathrm{Xu}^{\#}$, Youping $\mathrm{Wu}$, Wei Jiao \\ Department of Nursing, 904 Hospital of Joint Logistic Support Force of PLA, Wuxi, China \\ \#These authors contributed equally to this work. \\ Correspondence to: Wei Jiao. Department of Nursing, 904 Hospital of the Joint Logistics Support Force of the PLA, Wuxi, Jiangsu Province 214044, \\ China. Email: 4273187@qq.com.
}

\begin{abstract}
Intracranial inflammatory granuloma is a common intracranial occupying lesion. Common postoperative complications include intracranial edema, intracranial infection, hydrocephalus, epilepsy, and cerebrospinal fluid leakage. This report aims to summarize the nursing care of a patient with right frontoparietal inflammatory granuloma complicated with acute pulmonary embolism (APE). Acute pulmonary embolism is a clinical syndrome in which endogenous or exogenous emboli block the main trunk or branches of the pulmonary artery, resulting in disorders of pulmonary and respiratory circulation that seriously threatening the lives of patients. The occurrence and report of pulmonary embolism caused by intracranial inflammatory granuloma are rare. The patient had rapid onset, atypical clinical manifestations, and was in critical condition. Pulmonary embolism can easily lead to death. Nursing care after rapid thrombolysis included observation of coagulation function, prevention of complication, control of infection, improvement of intestinal dysfunction, maintenance and monitoring of sedation, prevention and observation of epilepsy, and prevention of the recurrence of embolism. After early intervention, active treatment and meticulous care, the patient's condition improved, mechanical ventilation was successfully withdrawn, and the patient was ultimately discharged and walked out on his own.
\end{abstract}

Keywords: Inflammatory granuloma; acute pulmonary embolism (APE); anti-infection; anticoagulation; antiepilepsy; prevention of recurrence of embolization

Submitted Apr 26, 2020. Accepted for publication Sep 16, 2020.

doi: 10.21037/apm-20-1479

View this article at: http://dx.doi.org/10.21037/apm-20-1479

\section{Introduction}

Intracranial inflammatory granuloma is a common intracranial occupying lesion. Common postoperative complications include intracranial edema, intracranial infection, hydrocephalus, epilepsy, and cerebrospinal fluid leakage, while the occurrence and documentation of pulmonary embolism are rare. Acute pulmonary embolism (APE) is a clinical syndrome in which endogenous or exogenous emboli block the main trunk or branches of the pulmonary artery, resulting in disorders of pulmonary and respiratory circulation that seriously threatening the lives of patients $(1,2)$. In this case of inflammatory granuloma, pulmonary embolism occurred after surgery. Esmon CT suggested (3) that inflammation promoted blood coagulation by inducing the expression of endovascular tissue factors, inducing the expression of white blood cell adhesion molecules on the surface of endovascular cells, and downregulating the anti-coagulation pathway of fibrinolytic protein and protein C. Pulmonary embolism lacks typical clinical manifestations, it is prone to missed diagnosis and misdiagnosis in clinical practice. Moreover, the onset of the disease is rapid, and if treatment is not timely, pulmonary embolism can easily lead to death. However, there is a lack of specific and targeted nursing measures in clinical 


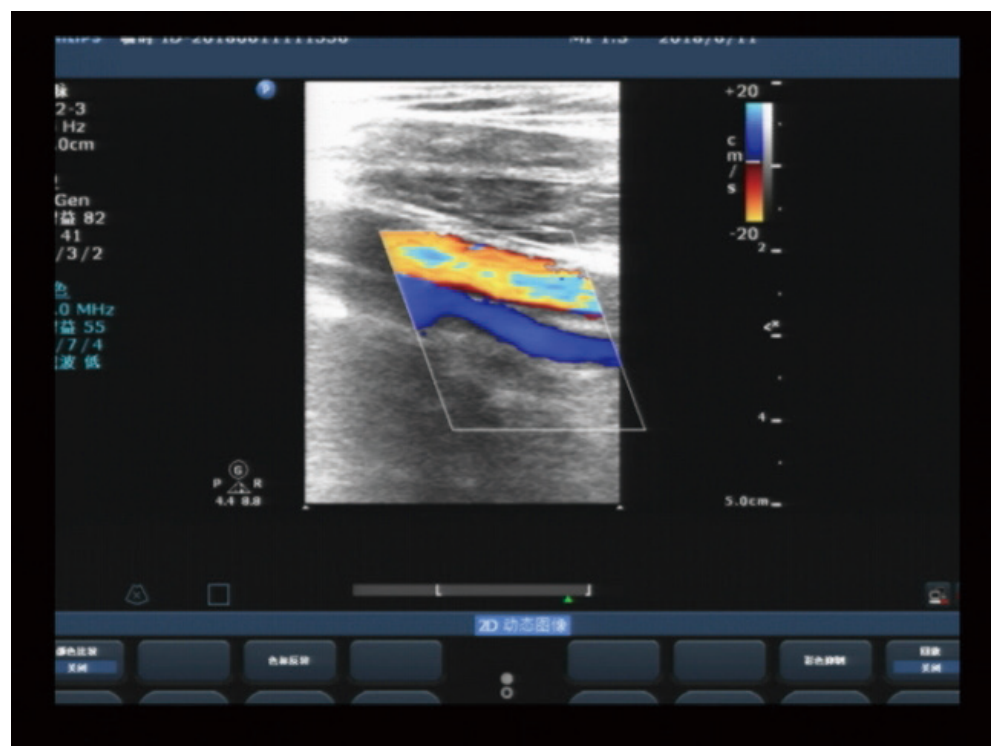

Figure 1 Scanning along the bilateral external iliac veins to their distal branches showed that the inner diameter of each vein was normal, and no clear abnormal echo was observed in their lumens. Color Doppler flow imaging showed good blood flow without significant abnormality.

practice. A patient with right frontoparietal inflammatory granuloma was admitted to the neurosurgery department of our hospital in May 2018 and developed pulmonary embolism on day 13 after granuloma resection. The nursing experience is reported below.

We present the following article in accordance with the CARE reporting checklist (available at http://dx.doi. org/10.21037/apm-20-1479).

\section{Clinical data}

A 59-year-old male patient was admitted to the emergency department on May 22, 2018, due to sudden disturbance of consciousness. He had no nausea, vomiting, or incontinence. His Glasgow Coma Scale (GCS) score was 15. His pupils were equal, round, and reactive to light. His muscle strength in all four limbs was grade 4, and muscle tension was normal. The patient had a history of hypertension. Head computed tomography (CT) showed right frontoparietal infarction with hemorrhage. After 15 days of treatment, the patient's symptoms were not significantly improved. After a further magnetic resonance angiography (MRA) examination, the patient was diagnosed with a right frontoparietal space-occupying lesion. After various preoperative examinations were completed, right frontotemporoparietal craniotomy for the resection of the space-occupying lesion was performed on June 13 . Postoperative pathology showed right frontoparietal inflammatory granuloma, which was believed to be caused by microorganisms. On the fifth day after the operation, the patient got out of bed and walked by himself. Reexamination of the complete blood count (CBC) showed that the white blood cell count was $12.42 \times 10^{9} / \mathrm{L}$, the neutrophil count was $9.52 \times 10^{9} / \mathrm{L}$, the neutrophil-to-lymphocyte ratio was 0.767 , C-reactive protein was $82.2 \mathrm{mg} / \mathrm{L}$, alanine aminotransferase was $66 \mathrm{U} / \mathrm{L}$, glutamine transferase was $62 \mathrm{U} / \mathrm{L}$, and the D-dimer level was high. Color Doppler ultrasound of the lower limb deep veins showed no definite abnormality (Figure 1). Considering that the patient had a large wound surface and was still in the perioperative period and that the use of anticoagulant drugs may cause delayed intracranial hemorrhage, ambulation was encouraged.

On June 26, the patient had sudden syncope, with a heart rate of 46 beats/min, respiratory rate of 6 breaths $/ \mathrm{min}$, and blood pressure $56 / 38 \mathrm{mmHg}(1 \mathrm{mmHg}=0.133 \mathrm{kPa})$. The patient's electrocardiogram showed rapid atrial fibrillation, complete right bundle branch block, and ST-segment elevation. Myocardial injury markers were checked immediately, and the myocardial zymograms were still normal. APE was considered, and computed tomography angiography (CTA) of the pulmonary artery was carried out immediately (Figure 2). The patient also 

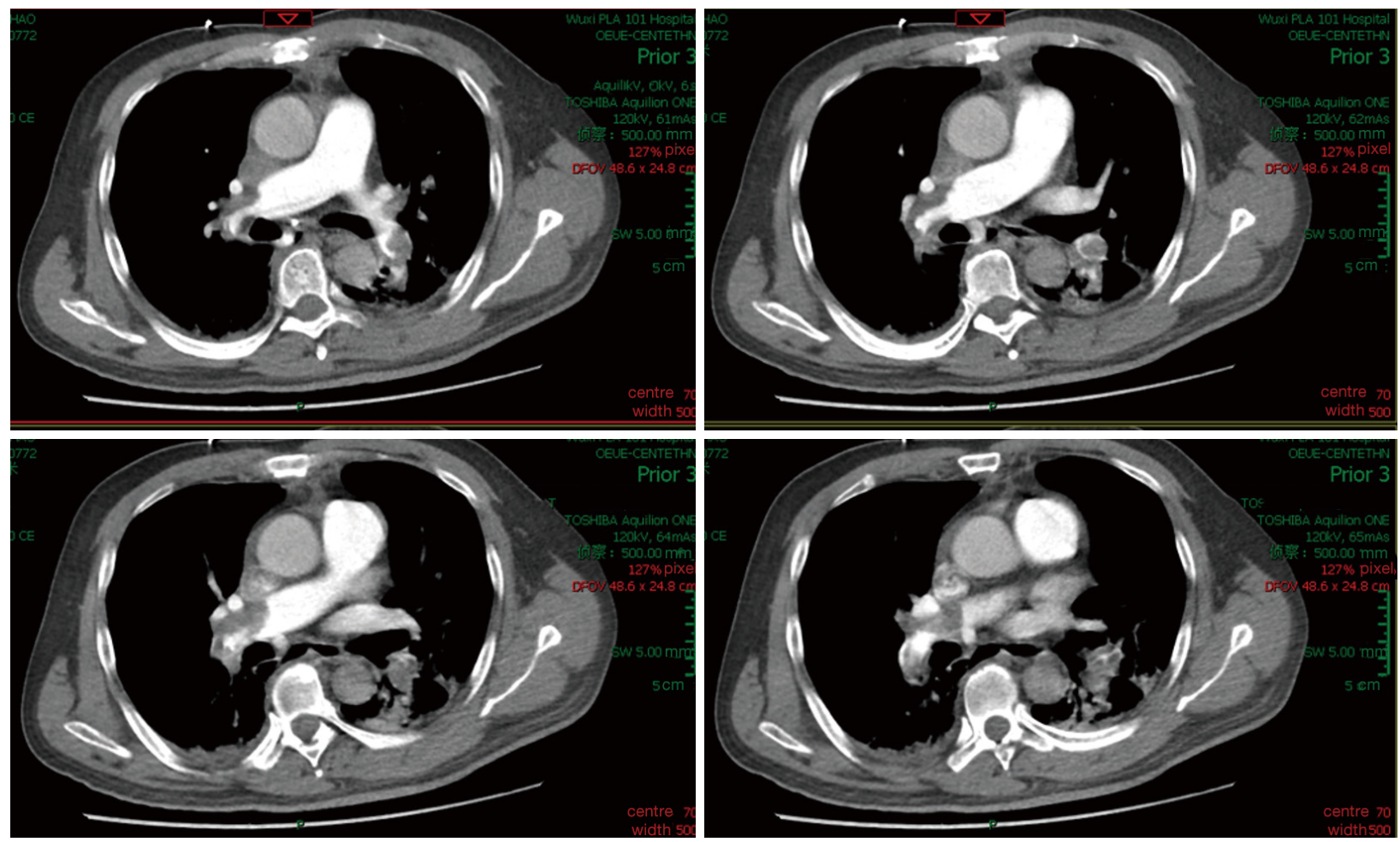

Figure 2 Multiple patchy low-attenuation areas were observed in the main trunk and branches of the pulmonary artery (indicated by arrows), suggesting bilateral pulmonary embolism.

received pulmonary artery intervention + inferior vena cava filter implantation + catheter-directed thrombolysis of the pulmonary artery and was then transferred to neurosurgical intensive care unit (NICU) for continuous treatment. After treatment, the patient was unconscious, his GCS score was 7, his pupils were equal and round, his heart rate was 120 beats/min, and his blood pressure was $100 / 60 \mathrm{mmHg}$. The patient was given mechanical ventilation to assist respiration and vasoactive drugs to maintain hemodynamic stability according to the doctor's advice. The CBC results were as follows: $\mathrm{pH}$ was 7.55 , base excess was 16.1 , actual bicarbonate was 38.5 , white blood cell count was $26.07 \times 10^{9} / \mathrm{L}$, neutrophil count was $23.58 \times 10^{9} / \mathrm{L}$, neutrophil-tolymphocyte ratio was 0.90 , alanine aminotransferase was $1,802 \mathrm{U} / \mathrm{L}$, glutamine transferase was $68 \mathrm{U} / \mathrm{L}$, D-dimer level was $99.99 \mathrm{mg} / \mathrm{L}$, and prothrombin time was $>160 \mathrm{~s}$. The lower limbs showed no swelling, the skin of the extremities was warm, and the dorsalis pedis artery was palpable. Color Doppler ultrasound of the lower limb deep veins performed on July 3 showed thrombosis in both lower limb deep veins (Figure 3). The infection had worsened, and chest CT showed atelectasis. The patient had no bowel movement for 3 consecutive days and had bowel sounds 3 times/min, and abdominal ultrasound showed flatulence. According to the doctor's advice, the patient received treatments for symptoms, including a sedative, a pressor agent, an anti-infective, an anticoagulant, liver protection agents, agents to improve gastrointestinal function, and agents for the prevention of epilepsy and recurrence of bleeding. At the same time, following rapid thrombolysis, nursing care that included observation of coagulation function, infection control, improvement of intestinal dysfunction, maintenance and monitoring of sedation, prevention and observation of epilepsy, and prevention of the recurrence of embolism was provided.

On July 16, the patient had clear consciousness, and his GCS score increased to 13 points. His pupils were equal, round, and reactive to light. His muscle strength in all four limbs was grade 5 , and his muscle tension was normal. The patient's hemodynamic status and liver function parameters tended to be stable, his spontaneous respiration gradually 


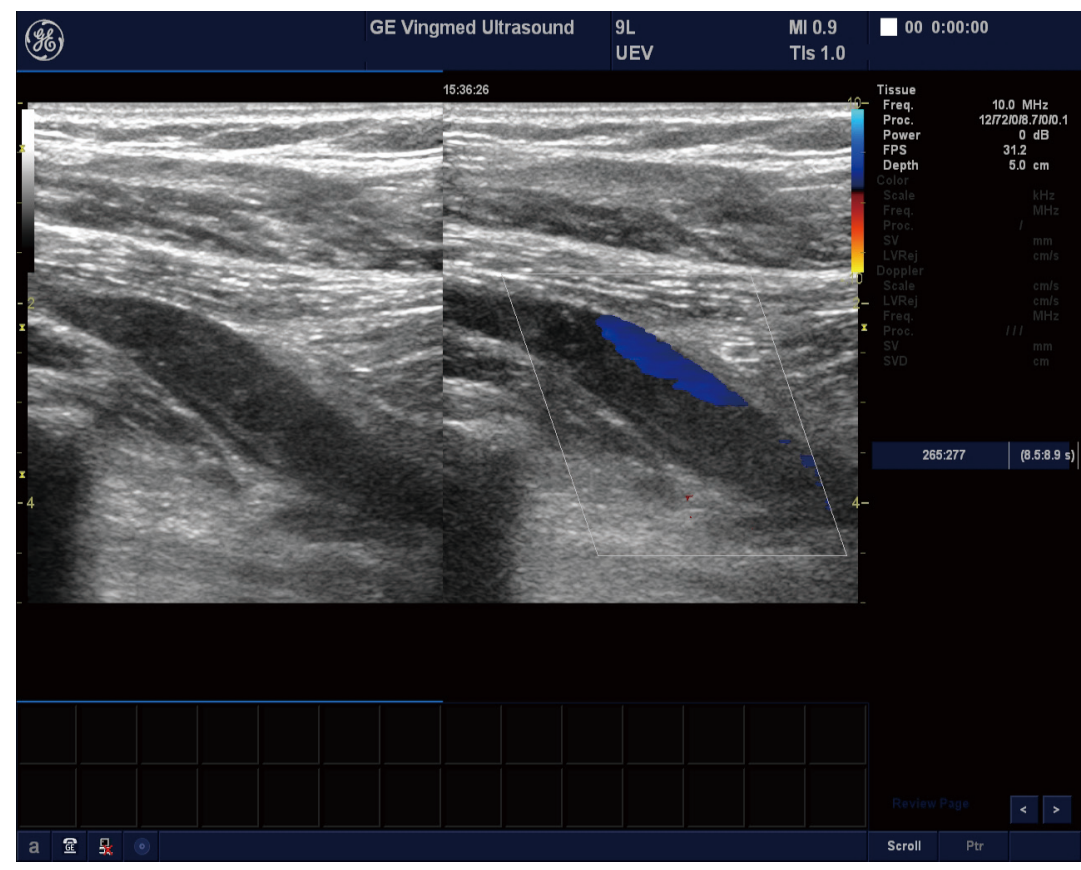

Figure 3 Thrombosis in the patient's right posterior tibial veins, left popliteal vein, and lower limb deep vein.

increased, the infection was effectively controlled, and the condition was improved. Mechanical ventilation was successfully withdrawn, and the patient was transferred to the general ward for continuous treatment. On July 29, color Doppler ultrasound reexamination of both lower limbs showed that the thrombus was significantly alleviated. On the 80th day after admission, the patient was discharged and walked out of the hospital by himself. The patient had a follow-up visit 3 months after the surgery, at which it was observed that he had recovered well and could take care of himself completely.

Written informed consent was obtained from the patient for publication of this study and any accompanying images. All procedures performed in studies involving human participants were in accordance with the ethical standards of the institutional and/or national research committee(s) and with the Helsinki Declaration (as revised in 2013).

\section{Nursing care}

\section{Anti-infective therapy}

The white blood cell count of cancer patients is associated with the risk of thrombosis, and neutrophils are a risk factor for venous thrombosis (4). The pathology of the patient's intracranial space-occupying lesion indicated inflammatory granuloma, and pulmonary and intracranial infection were present before and after the operation. The intracranial infection was effectively controlled after the operation, but the pulmonary infection was aggravated. Therefore, strengthening pulmonary care was the key point of nursing care. Based on the results of the patient's sputum culture, he was regularly given an appropriate amount of freshly prepared antibiotics for anti-infective therapy according to the doctor's advice, and the adverse reactions and effects of the medication were closely observed. Through routine turning once every hour, percussion on the back, and the use of a multifrequency vibrometer, mechanical ventilation and personalized airway care were strengthened. A high-flow oxygen concentrator was used to warm the air flow to $37^{\circ} \mathrm{C}$ and inject air with the maximum water content into the lung to provide continuous positive airway pressure to re-expand the alveoli, prevent alveolar atrophy and collapse, ensure the movement of lung cilia, and improve oxygenation (5). At the same time, prone position ventilation was provided. With the cooperation of four nurses, the patient was turned to the prone position, and the head was placed on a soft pillow to ensure that the airway was open for secretion drainage and improved oxygenation. After reasonable antiinfective treatment and nursing care were provided, the patient's pulmonary infection was effectively controlled, 
and the high-risk factors for the recurrence of pulmonary embolism were eliminated, which was conducive to the patient's recovery.

\section{Multiple initiatives to reduce the recurrence of pulmonary embolism}

\section{Observation and nursing care with anticoagulant therapy}

In terms of anticoagulant therapy for this patient with pulmonary embolism, acute enoxaparin of 4,000 IU was administered and maintained for 24 hours based on his body mass. Two days later, head and chest CT was repeated, and the patient still had thrombosis of the pulmonary artery. The amount of urokinase used was doubled, and pulmonary artery CTA was repeated. The greatest risk with anticoagulant thrombolysis is bleeding. During and within 2 hours after intravenous anticoagulant thrombolysis, the patient's blood pressure, pulse, vomiting, and consciousness were closely observed. At the same time, the puncture site was observed for exudation, hematoma, mucocutaneous bleeding, and other bleeding tendencies (6). CBC, bleeding and coagulation, liver and kidney function, and biochemistry were monitored daily. The D-dimer level was decreased from $25.93 \mathrm{mg} / \mathrm{L} \mathrm{FEU}$ to $7.81 \mathrm{mg} / \mathrm{L} \mathrm{FEU}$. On July 16, color Doppler ultrasound examination was repeated and showed that the patient's thrombosis was alleviated. The lower extremity catheter was removed. The intravenous thrombolysis was discontinued and replaced with subcutaneous injection of enoxaparin. Thrombin was dynamically monitored to evaluate the patient's coagulation function to prevent the occurrence of systemic hemorrhage, such as intracranial and retroperitoneal bleeding. Two weeks later, a repeat color Doppler ultrasound examination was performed and showed that some of the patient's vascular lumens were unobstructed. The anticoagulation regimen was changed to oral rivaroxaban tablets. The $\mathrm{D}$-dimer level was $2.72 \mathrm{mg} / \mathrm{L}$ FEU. The thrombus was effectively controlled, and no bleeding occurred during anticoagulation and thrombolysis.

\section{Progressive activity management}

In the acute period after the occurrence of pulmonary embolism in this patient, strict elevation and immobilization of both lower limbs were performed to prevent new embolisms caused by thrombus detachment. This patient had a high risk of thrombosis according to the Caprini risk assessment model. Graduated compression stockings that fit the circumference of both lower limbs of the patient were selected. Appropriate graded compression was used to improve the function of venous valves, regulate the levels of certain coagulation factors, and increase the oxygenation of the deep tissues of both lower limbs (7). The stockings were removed daily to assess limb condition, primarily skin color, skin temperature, dorsalis pedis pulse, numbness and pain in both lower limbs (8). Leg circumference was measured periodically, and a leg circumference curve was generated to facilitate handover and condition observation for each nursing shift. After the acute phase, massage and passive movement of both lower limbs were performed. A deep vein thrombosis pump was used 2 times/day for 30 min each to promote blood circulation. Bedside activities gradually transitioned to daily activities. The patient was asked to avoid sitting or standing for a long time and to avoid strenuous activities. Good skin care was provided for the patient to prevent skin damage.

\section{Improvement of gastrointestinal function}

The monitoring of the intestinal mucosal barrier was strengthened. The patient's intestinal mucosa was mildly damaged, and parenteral nutrition was gradually reduced until the patient's gastrointestinal function was effectively improved. Early enteral nutrition care was provided. An enteral feeding pump was used to infuse Peptisorb (Nutricia) and a glucose-saline solution. The rate of infusion was adjusted dynamically according to the patient's tolerance. The gastric residues were aspirated every 4 hours to observe the volume, color, and property of the gastric juice, and abdominal distension, nausea, and vomiting were assessed using the Enteral Nutrition Support Tolerance Assessment Form to prevent the exacerbation of pulmonary infection caused by accidental aspiration. Bowel sounds were auscultated every 2 hours, abdominal massage was performed, and stool conditions were observed. Prokinetic agents were also added to increase gastrointestinal propulsive motility, enhance gastrointestinal contractility, promote and stimulate gastrointestinal emptying, and avoid the formation of new emboli from shed thrombi due to straining to have a bowel movement. Through the assessment of early enteral nutritional support and gastrointestinal function, the patient's nutritional status was good and all indexes were within the normal range.

\section{Prevention of epilepsy}

Epilepsy is often the first clinical symptom of granulomatous changes. After surgery, a mixture of $400 \mathrm{mg}$ 
of sodium valproate injection and $50 \mathrm{~mL}$ of normal saline was continuously infused by a micropump at a rate of $4 \mathrm{~mL} /$ min to prevent epilepsy, and the concentration of sodium valproate was monitored regularly. Since the patient had acute liver injury due to APE and sodium valproate is highly toxic to the liver, it was discontinued and replaced with oral levetiracetam tablets for the prevention of epilepsy. Safety measures for epilepsy were taken to prevent injury and redetachment of the emboli during grand mal seizures. The patient had no epileptic seizures during treatment.

\section{Observation and first aid of cardiac arrest}

The patient with pulmonary embolism was given 24hour ecg monitoring and special care. Rescue vehicle, defibrillator and endotracheal intubation should be placed beside the bed for standby use. To observe the clinical manifestations such as sweating, irritability, dyspnea, and chest tightness in the prodrome and onset of cardiac arrest. In case of cardiac arrest, external chest compression, endotracheal intubation, intravenous cardiac stimulant, respiratory stimulant and other rescue drugs should be given immediately, and electric shock defibrillation should be given in the early stage. The doctor in charge should inform the interventional department to take thrombus treatment immediately.

\section{Maintenance and monitoring of analgesia and sedation levels}

Studies have shown $(9,10)$ that continuous light sedation does not inhibit respiration and circulation but encourages awakeness in patients, facilitates nurses' ability to determine whether patients can be weaned and extubated, and reduces the incidence of ventilator-associated pneumonia. The patient had a pain score of 7 on admission and GCS dropped to 7 after pulmonary embolism. While the patient was unconscious and under mechanical ventilation, $3 \mathrm{mg}$ of remifentanil combined with 200 micrograms of dexmedetomidine were intravenously infused by a micropump for sedation and analgesia. According to the pain assessment table, pain was maintained at $0-4$ points. The bispectral index (BIS) was used to monitor the depth of sedation and was maintained at $60-80$ points. The drug infusion rate was strictly controlled to ensure that the smallest dose possible was used and prevent a large amount of sedative drug from entering the patient's system in a short time and causing adverse reactions. The degree of analgesia and sedation was scored at every shift to prevent abnormalities such as hypotension and hemodynamic instability caused by the use of analgesic and sedative drugs. Effective analgesia and sedation can significantly improve the comfort level of patients.

\section{Nursing for the liver injury}

This patient with pulmonary embolism had acute liver injury after surgery and was given polyene phosphatidylcholine injection combined with magnesium isoglycyrrhizinate injection according to the doctor's advice. Attention was paid to the observation of and nursing care for the patient's complications, especially dangerous complications such as hepatic encephalopathy and gastrointestinal bleeding, and preparations were made to provide rescue at any time. Magnesium isoglycyrrhizinate injection may cause pseudoaldosteronism, i.e., hypokalemia, and elevated blood pressure, and water and sodium retention may occur after medication (11). Therefore, changes in the patient's vital signs, electrolytes, and liver function were closely monitored, and medication was given only when definitive indications were observed. The patient's family members were educated about the characteristics of the disease and about safe medication to address and reduce their tension and anxiety.

\section{Psychological care}

After the patient was admitted to the hospital and finally diagnosed with intracranial inflammatory granulomas, he and his family members were given a simple and understandable explanation of and information about the condition, and additional care was provided according to their psychological needs. However, after the patient developed pulmonary embolism, his family members feared that the development of the disease would threaten the patient's life, so increased psychological guidance was provided. The Hamilton Anxiety Scale (HAMA) and a family member interview were used to evaluate the degree of anxiety. The members of the psychological nursing professional group in the department provided psychological guidance and explained the significance of thrombolysis, preoperative preparation, potential postoperative complications and preventive measures, the importance and necessity of various treatment and nursing measures for the patient, and the precautions being taken in the intensive care. As a result, the family members' 
confidence in the medical staff and their sense of security were increased, their anxiety and fear were alleviated, and they were able to actively cooperate with the treatment plan that the medical staff had developed for the patient to avoid the progression of the disease.

\section{Supervision of and feedback on nursing quality}

A system of assigning a specific person to each critically ill patient was used, and an Observation and Verification Sheet for Critically Ill Patients was developed. For key and difficult problems in care for this patient, each shift conducted a systematic assessment and handover process. The nursing group leader regularly examined the development of the patient's condition, tracked it, and provided feedback on the quality of nursing services to promote continuous improvement. Weekly disease reporting meetings of difficult cases were held to present disease reports and progress evaluations and to discuss any problems that the responsible nurses were experiencing. Nursing brainstorming sessions were carried out to help the nurses in the department master the ability to discuss and analyze difficult problems, learn independently, and handle practical problems.

\section{Discussion}

The incidence of inflammatory granulomas in the brain is low, and its imaging features are characteristic, generally small, with neat edges. The lesions on plain CT showed equal or slightly high density with rare calcification (12). It still needs to be differentiated from brain abscess, glioma, meningioma and so on. Brain abscesses mostly have systemic or local infection symptoms, with obvious mass effect, severe peripheral edema, and even and significantly enhanced wall thickness. Glioma often shows edge irregular bump, occur in medullary area more, with irregular or incomplete ring enhancement more common, without meninges enhancement, edema around tumor obvious, significant occupying effect, such as brain tissue, ventricle and brain cisterna compression deformation. Meningiomas are mostly connected with the meninges in a broad base, with higher density. After enhancement, meningiomas are significantly enhanced uniformly. Meningiomas often have cauda meningiomas and can be accompanied by local cranial reactions.

This patient with an inflammatory granuloma developed a postoperative pulmonary embolism after surgery, possibly due to the secondary inflammation and clotting reaction caused by systemic infection. Inflammatory cytokines are able to activate coagulation, and inflammation and coagulation can jointly lead to intravascular injury and microvascular thrombosis (13). Clinical nurses should strengthen the ability to provide a timely evaluation of thrombotic risk factors and should improve and develop various preventive measures according to the evaluation results to avoid thrombosis and pulmonary embolism.

In today's clinical treatment measures are more diversified, the use of sedation measures makes patients more comfortable and conducive to the smooth progress of various treatments; Individualized activity management and anticoagulant therapy played a key role in the prevention and treatment of pulmonary embolism. The management of gastrointestinal tract and the prevention of epilepsy played a good role in promoting the smooth recovery of patients. Effective psychological nursing increases the confidence of patients and their families in medical staff and ensures the smooth development of nursing work.

Personalized care should be developed for patients through an evidence-based approach while observing and recording changes in the patient's condition. In addition, anticoagulation, anti-infection, liver protection, sedation, and epilepsy prevention care should be provided according to the doctor's advice. Respiratory and circulatory support should be well prepared, and progressive activity of the patient should be managed to reduce the risk of recurrence of pulmonary embolism and improve the prognosis. However, there are limitations in our nursing study. This study is a case study, involving rare cases. Therefore, there are many potential benefits for better nursing research if more samples are available.

\section{Acknowledgments}

Funding: None.

\section{Footnote}

Reporting Checklist: The authors have completed the CARE reporting checklist. Available at http://dx.doi.org/10.21037/ apm-20-1479

Conflicts of Interest: All authors have completed the ICMJE uniform disclosure form (available at http://dx.doi. org/10.21037/apm-20-1479). The authors have no conflicts of interest to declare. 
Ethical Statement: The authors are accountable for all aspects of the work in ensuring that questions related to the accuracy or integrity of any part of the work are appropriately investigated and resolved. Written informed consent was obtained from the patient for publication of this study and any accompanying images. All procedures performed in studies involving human participants were in accordance with the ethical standards of the institutional and/or national research committee(s) and with the Helsinki Declaration (as revised in 2013).

Open Access Statement: This is an Open Access article distributed in accordance with the Creative Commons Attribution-NonCommercial-NoDerivs 4.0 International License (CC BY-NC-ND 4.0), which permits the noncommercial replication and distribution of the article with the strict proviso that no changes or edits are made and the original work is properly cited (including links to both the formal publication through the relevant DOI and the license). See: https://creativecommons.org/licenses/by-nc-nd/4.0/.

\section{References}

1. Zhang M, Wang N, Zhai Z, et al. Incidence and risk factors of chronic thromboembolic pulmonary hypertension after acute pulmonary embolism: a systematic review and meta-analysis of cohort studies. J Thorac Dis 2018;10:4751-63.

2. Rawal A, Ardeshna D, Hesterberg K, et al. Is there an optimal "door to cath time" in the treatment of acute pulmonary embolism with catheter-directed thrombolysis? Ann Transl Med 2019;7:419.

3. Esmon CT, Taylor FB, Jr, Snow TR. Inflammation and coagulation: linked processes potentially regulated through a common pathway mediated by protein C. Thromb Haemost 1991;66:160-5.

4. Blix K, Jensvoll H, Brækkan SK, et al. White blood cell count measured prior to cancer development is associated with future risk of venous thromboembolism--the Tromsø study. PLoS One 2013;8:e73447.

Cite this article as: Zhang J, Xu Y, Wu Y, Jiao W. Nursing care for a patient with right frontoparietal inflammatory granuloma complicated with acute pulmonary embolism. Ann Palliat Med 2020;9(5):3643-3650. doi: 10.21037/apm-20-1479
5. Fang T, Jiang H, Xu Y, et al. Evaluation of the effect of nasal high-flow oxygen therapy for the sequential treatment of mechanical ventilation and weaning. Journal of Cardiovascular and pulmonary Diseases 2019;38:673-6.

6. Wang Y, Chao X, Yan X, et al. Nursing care of patients with superior mesenteric vein thrombosis treated by transarterial thrombolysis. Journal of Nurse Training 2019;34:156-8.

7. Professional Committee on Nursing Care, Chinese Chapter Congress of the International Union of Angiology; Society of Endovascology, Chinese Medical Doctor Association. Expert consensus on the application of graduated compression stockings for prevention and treatment of venous thromboembolism. Journal of Interventional Radiology 2019;28:811-8.

8. Tian X, Fang W. Thrombolytic therapy in cardiopulmonary resuscitation for patients with sudden cardiac arrest caused by pulmonary embolism. Chinese Journal of Tuberculosis and Respiratory Diseases 2014;373:219-20.

9. Wu Y, Gong H, Gao Y. Application of a goal-oriented scheme for continuous mild sedation in patients with mechanical ventilation. Chinese Journal of Nursing 2017;52:400-4.

10. Turunen H, Jakob SM, Ruokonen E, et al. Dexmedetomidine versus standard care sedation with propofol or midazolam in intensive care: an economic evaluation. Crit Care 2015;19:67.

11. Hou L, Ren L, Wang Z, et al. Observation of the protective effect of magnesium isoglycyrrhizinate injection on liver function in patients with acute liver injury. Shanxi Medical Journal 2017;46:1484-6.

12. Zhang D, Han X, Meng H, et al. CT analysis of intracranial inflammatory granuloma (a report of 44 cases). Journal of Practical Radiology 2000;16:700-1.

13. Allegrini D, Reposi S, Nocerino E, Pece A. Odontogenic orbital cellulitis associated with cavernous sinus thrombosis and pulmonary embolism: a case report. J Med Case Rep 2017;11:164. 